\title{
Frecuencias e indicadores de riesgo de las recesiones gingivales en una muestra peruana
}

\section{Frequencies and risk indicators of gingival recession in a Peruvian simple}

\author{
Yuri Castro-Rodríguez ${ }^{1^{*}}$, Sixto Grados-Pomarino²
}

1. Cirujano Dentista, Facultad de Odontología, Universidad Nacional Mayor de San Marcos, Lima, Perú.

2. Especialista en Periodoncia, Coordinador de la especialidad de Periodoncia de la Facultad de Odontología, Universidad Nacional Mayor de San Marcos, Lima, Perú.

*Correspondencia a: Yuri Castro-Rodríguez | E-mail yuricastro16@hotmail.com

Trabajo recibido el 10/04/2016. Aprobado para su publicación el 11/09/2016

\section{RESUMEN}

Objetivo: Evaluar la prevalencia, extensión, severidad e indicadores de riesgo de recesiones gingivales en Lima, Perú.

Materiales y métodos: Una muestra representativa de 318 adultos entre 25-75 años fueron evaluados. Se formuló un cuestionario estructurado y un examen clínico en la zona vestibular de todas las piezas dentarias. La recesión gingival fue evaluada por sujetos de estudio, por piezas dentarias y por la profundidad de cada una de ellas (promedio). Los indicadores de riesgo evaluados fueron: nivel de educación, género, estado socioeconómico, movimiento del cepillado, frecuencia de cepillado, frecuencia del uso de hilo dental y los tratamientos periodontales y ortodóncicos previos. El modelo de regresión multivariada fue utilizado para establecer las asociaciones.

Resultados: La prevalencia de recesiones gingivales fue del 94,3\%, la extensión fue del $44,8 \%$, siendo más comunes en la región mandibular $(54,2 \%)$; la severidad promedio de las recesiones fue de $3,86 \pm 0,45 \mathrm{~mm}$. El género masculino evidenció una asociación significativa con la severidad de las recesiones (OR=2; IC: 1,09-3,68).

Conclusión: Las recesiones gingivales son altamente prevalentes en una población peruana. Los principales indicadores de riesgo encontrados fueron el género, el movimiento del cepillado y el tratamiento ortodóncico previo.

\section{PALABRAS CLAVE}

Perú; Recesión gingival; Epidemiología; Prevalencia; Indicadores de riesgo

Rev. Clin. Periodoncia Implantol. Rehabil. Oral Vol. 10(3); 135-140, 2017.

\section{ABSTRACT}

Objective: To evaluate the prevalence, extent, severity and risk indicators of gingival recession in a population of Lima, Peru.

Materials and methods: A representative sample of 318 adults aged 25-75 years was evaluated. A structured questionnaire was completed, and a clinical examination performed in the vestibular area of all teeth. Gingival recession was assessed by study subject, by tooth and depth of each (mean). The risk indicators assessed were: level of education, gender, socioeconomic status, brushing movement, brushing frequency, frequency of flossing, and previous periodontal and orthodontic treatments. A multivariate regression model was used to establish associations.

Results: The prevalence of gingival recession was $94.3 \%$, and the extension was $44.8 \%$, being more common in the mandibular region $(54.2 \%)$. The mean severity of recessions was $3.86 \pm 0.45 \mathrm{~mm}$. Males showed a significant association with the severity of recessions ( $\mathrm{OR}=2, \mathrm{Cl}$ : 1.09-3.68).

Conclusion: Gingival recessions were highly prevalent in this Peruvian population. The main risk indicators found were gender, brushing movement, and previous orthodontic treatment.

KEYWORDS

Perú, Gingival recession; Epidemiology; Prevalence; Risk indicators

Rev. Clin. Periodoncia Implantol. Rehabil. Oral Vol. 10(3); 135-140, 2017. 


\section{INTRODUCCIÓN}

La recesión gingival $(R G)$ es el desplazamiento del margen gingival apical a la unión del cemento y esmalte, con la exposición de la superficie radicular al ambiente oral(1). Es una condición frecuente tanto en pacientes con buena y mala higiene oral ${ }^{(2)}$, así como en pacientes con enfermedad periodontal incipiente 0 enfermedad avanzada(3). Las principales quejas que ocasiona en los pacientes son los problemas estéticos y de hipersensibilidad, siendo esta última un factor que dificulta la remoción de placa bacteriana en la zona del defecto ${ }^{(3)}$; también son comunes los problemas de caries radicular, molestias y mayor susceptibilidad a la inflamación ${ }^{(4)}$.

La RG es una condición periodontal que se presenta muy frecuentemente en los pacientes. Esta puede ser considerada como una manifestación clínica de pérdida de inserción periodontal. De acuerdo con Maetahara ${ }^{(5)}$ la prevalencia de recesiones es del $72,9 \%$ en una población peruana. Susin ${ }^{(6)}$ acota una prevalencia de $83,4 \%$ en una población brasileña. También se ha reportado una prevalencia de $27 \%$ en pacientes entre $18-25$ años $^{(7)}$, del $62 \%$ en pacientes entre $16-25$ años $^{(8)}$ y del $100 \%$ en pacientes entre $46-86$ años $^{(9)}$. Uno de los estudios de mayor consideración es el de Albandar y Kingman ${ }^{(10)}$, quienes en 23,8 millones de personas norteamericanas encuentran una prevalencia de $58 \%$, con un promedio de $3 \mathrm{~mm}$ de profundidad de la RG, acotan además que existe una correlación positiva entre la extensión y severidad de la RG con el aumento de la edad, siendo más comunes en la superficie vestibular y en el género masculino.

Novaes $^{(11)}$ considera que la principal causa de las recesiones gingivales son la placa bacteriana y el traumatismo mecánico; sin embargo, se considera que la etiología es multifactorial y siempre resulta de la interacción de varios de ellos: anatómicos (dehiscencia del hueso alveolar, inserción elevada de músculos, traumatismo oclusal, tensión del frenillo, biotipo gingival), inflamatorios (enfermedad periodontal destructiva, presencia de placa dental y cálculo supra/infragingival, inadecuado cepillado dentario), factores traumáticos (vigorosos hábitos de higiene oral, piercing oral) y factores iatrogénicos ${ }^{(8,12-14)}$. El fumar es considerado como uno de los factores de riesgo ${ }^{(6)}$; otros indicadores de riesgo incluyen la edad, el género masculino, la raza y la condición de fumador ${ }^{(6,9,15)}$. Existe poca información sobre la asociación entre RG y otros factores como la historia periodontal, el tratamiento ortodóncico la inflamación gingival y las prácticas de higiene oral ${ }^{(16)}$. La presente investigación tuvo como objetivo cuantificar la prevalencia, extensión y severidad de las recesiones gingivales en una población peruana.

\section{MATERIALES Y MÉTODOS}

\section{Población de estudio}

Estudio transversal, observacional, realizado en la Facultad de Odontología de la Universidad Nacional Mayor de San Marcos. Incluyó a los pacientes atendidos en el servicio de Periodoncia desde inicios de año 2014 hasta fines del año 2015. Población de 357 pacientes en su totalidad

\section{Consideraciones éticas}

El protocolo de estudio fue evaluado y revisado por el Instituto de Investigación de la Facultad de Odontología. Antes de cada encuesta los pacientes evaluados firmaron un consentimiento informado junto con el desarrollo de la historia clínica.

\section{Tamaño de la muestra}

Considerando que el estudio evaluó varias variables dentro de la cavidad bucal se estimó un $50 \%$ de prevalencia para cada condición. Se consideró una precisión del $4 \%$ y un $95 \%$ de intervalo de confianza. Acorde a la fórmula estándar para la estimación de prevalencias, y según el ajuste de la muestra, se necesitaron 210 pacientes.

\section{Estudio de la muestra}

La mayoría de la población fue encuestada y evaluada clínicamente, algunos sujetos fueron excluidos por su condición de edentulismo total por su corta edad (menores de 20 años) y por su no aceptación con la participación en el estudio. En total fueron encuestados y evaluados 319 pacientes para el análisis de datos.

\section{REGISTRO DE DATOS}

\section{Encuesta}

Los pacientes fueron encuestados a través de un cuestionario estructurado, y este incluyó datos sociodemográficos, hábitos de higiene oral, accesos a los servicios dentales, historia médica y hábitos de consumo. Dos asistentes entrenados y calibrados fueron los encargados de recabar esta información. El nivel de educación fue definido acorde a la cantidad de años de educación: baja ( $\leq 4 a n ̃ o s)$, media (5-10 años), alta (>10años). El nivel socioeconómico a través de la CCEB Clasificación ${ }^{(17)}$ que considera la cantidad de consumos y nivel de educación de la cabeza de familia como baja ( $\leq 20$ puntos), media (21-26 puntos) y alta (>26 puntos). El tipo de cepillado fue categorizado como horizontal, vertical, circular y como combinación entre ellos. La frecuencia del cepillado fue categorizado como $\leq 1$ vez/ día, 2 veces/día y $\geq 3$ veces/ día. La frecuencia de limpieza interproximal como nunca, $\leq 1 \mathrm{vez} /$ día y $\geq 2$ veces/día. La exposición al tabaco fue evaluada como la cantidad de cigarrillos consumidos en un día, por el número de años de hábito y divididos entre 20; así se categorizó como: no fumadores (0 cajetillas por año), fumador moderado ( $<20$ cajetillas por años) y fumador severo ( $\geq 20$ cajetillas por año). La frecuencia de visita dental fue categorizada como regular si acudían $\geq 1$ vez/año; las visitas realizadas por urgencias y/o emergencias fueron catalogadas como visitas irregulares.

\section{Examen clínico}

Se analizaron todas las piezas dentarias totalmente erupcionadas, 2 examinadores realizaron la evaluación con una sonda periodontal (North Carolina de $15 \mathrm{~mm}$ ), la cantidad de placa bacteriana fue evaluada en todas las caras de las piezas dentarias a través del índice de higiene oral de O'Leary; la presencia de recesiones gingivales fue evaluada en la superficie vestibular. La RG fue definida como la distancia desde la unión cemento esmalte hasta el margen gingival libre; si la unión cemento esmalte se encontró apical al margen gingival se consideró como una medida negativa. La severidad de la enfermedad gingival fue evaluada a través de la cantidad de zonas de sangrado al sondaje y categorizadas como baja $(\leq 22 \%)$ y alta $(>22 \%)$. La profundidad de la RG fue categorizada como ausente $(0 \mathrm{~mm}), \geq 1 \mathrm{~mm}, \geq 3 \mathrm{~mm}$ y $\geq 5 \mathrm{~mm}$.

\section{Reproductibilidad}

La confiabilidad del cuestionario fue realizada a través del estudio piloto con 50 pacientes y utilizando la prueba y reprueba. El coeficiente Kappa para las preguntas osciló entre 0,91-0,99. La confiabilidad intra e interexaminador del examen clínico de las recesiones gingivales también fue realizada durante el estudio piloto, un total de 16 pacientes fueron incluidos (448 sitios). La confiabilidad intraexaminador para los datos categóricos varió entre 0,91-0,99 (kappa), el análisis interexaminador para la profundidad de las recesiones gingivales fue de 0,84 (coeficiente de correlación intraclase).

\section{Análisis estadístico}

La prevalencia de recesiones gingivales fue definida como el porcentaje de individuos que presentaban al menos un diente con RG. La extensión de las recesiones fue definida como el porcentaje de piezas dentarias con recesiones gingivales. La severidad de la RG fue analizada utilizando el promedio individual de la recesión; los datos del cuestionario fueron evaluados a través de un análisis de regresión multivariado para determinar su asociación con la RG. Un proceso de 2 etapas se utilizó para investigar la influencia de los posibles factores de riesgo y los resultados de recesiones gingivales; en primer lugar se utilizó el análisis bivariante para poner a prueba la relación entre las recesiones gingivales y los factores asociados, además se utilizó los odds ratio con un $95 \%$ de intervalo de confianza para evaluar las relaciones bivariadas entre las variables examinadas; una odds ratio ajustada, con IC al 95\% también fue utilizada. Para la asociación entre la variable dependiente y las variables independientes se utilizó el modelo de regresión logística multivariable. El modelo de regresión lineal fue evaluado a través del análisis de los residuos; la multicolinealidad y las interacciones no fueron encontradas durante la correlación de variables. Se aceptó una significación del $0,05 \%$.

\section{RESULTADOS}

Del total de la muestra evaluada (318 sujetos), 300 (94,3\%) evidenciaron por lo menos una recesión $\geq 1 \mathrm{~mm}$ en al menos un diente; 18 sujetos $(5,7 \%)$ no presentaron ninguna RG. El promedio de edad de los sujetos fue de $42 \pm 16,25$, el $47,2 \%$ fueron varones, el $52,8 \%$ fueron mujeres. Excluyendo las piezas dentarias ausentes en la muestra fueron evaluados 6.890 dientes; $3.808(44,8 \%)$ evidenciaron al menos recesiones gingivales $\geq 1 \mathrm{~mm}, 2.064(54,2 \%)$ al nivel de la mandíbula y $1.744(45,8 \%)$ al nivel del maxilar.

En lo que respecta a los sujetos, la prevalencia de recesiones gingivales entre 1-2mm fueron las más encontradas (58\%), siendo lo más prevalente en el grupo de personas entre los 50-59 años de edad (fig. 1). La extensión de las recesiones gingivales abarcó en un $78 \%$ a recesiones de $1-2 \mathrm{~mm}$. La severidad promedio de las recesiones gingivales fue de $3,86 \pm 0,45 \mathrm{~mm}$, siendo este valor progresivo en cuanto a los estratos por edad (tabla 1).

El género femenino evidenció mayor prevalencia de recesiones gingivales (49,1\% vs $45,3 \%$ ) (fig. 2 ). Una mayor tasa para los pacientes con un nivel de educación alto $(54,1 \%)$ y para pacientes con un estado 
Tabla 1. Prevalencia (porcentaje por sujeto), extensión (por pieza dentaria) y severidad (promedio de la recesión gingival) acorde a los grupos de edad y considerando la superficie vestibular

\begin{tabular}{|c|c|c|c|c|c|c|c|c|}
\hline & \multicolumn{8}{|c|}{ Edad } \\
\hline & \multirow{2}{*}{$\frac{20-29}{n}$} & \multirow{2}{*}{$\begin{array}{c}30-39 \\
n\end{array}$} & \multirow{2}{*}{$\begin{array}{c}\text { 40-49 } \\
n\end{array}$} & \multirow{2}{*}{$\begin{array}{c}50-59 \\
n\end{array}$} & \multirow{2}{*}{$\begin{array}{c}60-69 \\
n\end{array}$} & \multirow{2}{*}{$\frac{>70}{n}$} & \multicolumn{2}{|c|}{ Total } \\
\hline & & & & & & & $\mathbf{N}$ & $\%$ \\
\hline \multicolumn{9}{|l|}{ Prevalencia } \\
\hline $\mathrm{RG} \geq 1 \mathrm{~mm}$ & 44 & 40 & 64 & 84 & 60 & 20 & 312 & 58 \\
\hline $\mathrm{RG} \geq 3 \mathrm{~mm}$ & 6 & 12 & 34 & 52 & 46 & 16 & 166 & 31 \\
\hline $\mathrm{RG} \geq 5 \mathrm{~mm}$ & 0 & 2 & 14 & 20 & 14 & 8 & 58 & 11 \\
\hline \multicolumn{9}{|l|}{ Extensión } \\
\hline $\mathrm{RG} \geq 1 \mathrm{~mm}$ & 332 & 340 & 728 & 838 & 588 & 130 & 2956 & 78 \\
\hline$R G \geq 3 m m$ & 8 & 26 & 168 & 238 & 198 & 64 & 702 & 18 \\
\hline $\mathrm{RG} \geq 5 \mathrm{~mm}$ & 0 & 20 & 28 & 46 & 44 & 12 & 150 & 4 \\
\hline Severidad & $1,7 \pm 0,8$ & $2,4 \pm 0,4$ & $2,9 \pm 0,1$ & $3,8 \pm 1,7$ & $4,1 \pm 0,8$ & $4,4 \pm 1,12$ & $3,86 \pm 0,7$ & $3,86 \pm 0,45$ \\
\hline
\end{tabular}

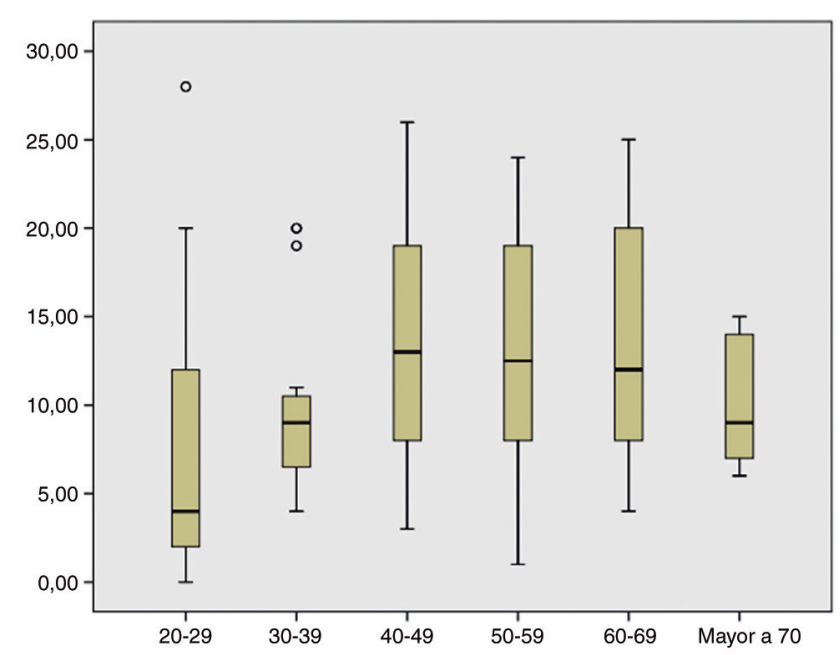

socioeconómico medio (72,3\%). Una frecuencia de cepillado de 2 veces/ día, no utilizar hilo dental y aquellos que realizaban una técnica de cepillado en sentido vertical evidenciaron mayores tasas de recesiones gingivales $(50,3 \%, 72,2 \%$ y $35,2 \%$ respectivamente). Una mayor cantidad de recesiones fue encontrada en pacientes no fumadores $(81,8 \%)$; sin embargo, de los fumadores la totalidad de los mismos evidenció al menos una recesión gingival $\geq 1 \mathrm{~mm}$. Pacientes que no recibieron tratamiento periodontal y/o ortodóncico previo evidenciaron una mayor cantidad de recesiones gingivales (tabla 2 ).

Se encontró asociación entre el género masculino y la severidad de las recesiones gingivales, siendo significativo para las recesiones de $3-4 \mathrm{~mm}$ $y \geq 5 \mathrm{~mm}$ (OR=1,6, IC: 1,05-2,5 y OR=2, IC: 1,09-3,68, respectivamente). El movimiento del cepillado circular y las recesiones de $3-4 \mathrm{~mm}$ y $\geq 5 \mathrm{~mm}$ también encontraron asociaciones significativas (OR=0,51, IC: 1,19-1,35, $\mathrm{OR}=0,78, \mathrm{IC}=1,14-4,27$, respectivamente). El tratamiento ortodóncico previo presentó una asociación con las recesiones $\geq 5 \mathrm{~mm}$; $\mathrm{OR}=1,2$; IC=1,14-1,27 (tabla 3).

\section{DISCUSIÓN}

La etiología básica de una RG es la inflamación gingival, la cual puede ser causada por el cúmulo de placa bacteriana o mecánicamente (cepillado traumático); sin embargo, la asociación entre el cepillado y la aparición de recesiones gingivales y lesiones cervicales no cariosas aún es inconclusa(18), y sí se ha encontrado que pacientes con cepillado manual mecánico tienen mayor cantidad de recesiones gingivales luego de 12 meses de uso que los que utilizan cepillos eléctricos ${ }^{(18)}$. El principal factor del cepillado asociado con la aparición de recesiones es la frecuencia y el método(18). La asociación entre el movimiento del cepillado y la severidad de las recesiones gingivales solo fue significativa para aquellos pacientes con movimientos circulares $(\mathrm{OR}=0,51$ y $\mathrm{OR}=0,78)$; también se encontró asociaciones entre la frecuencia del cepillado y la severidad, sin embargo esta no fue significativa $(p>0,05)$.

La prevalencia de recesiones aumenta con la edad, pero no es una consecuencia de la misma ${ }^{(19)}$. Aparecen incluso en personas con buena higiene oral, y sus causas son multifactoriales, el cepillado es una de ellas. Se ha demostrado que la edad es un indicador de riesgo fuerte para la aparición de recesiones ${ }^{(6,10,16)}$, en la muestra evaluada la mayor prevalencia de recesiones fue encontrada entre los 50-59 años, siendo menor la tasa en pacientes jóvenes (20-29 años). Esto puede explicarse por la experiencia acumulativa de pérdida de soporte periodontal y exposición a factores de riesgo durante la vida ${ }^{(16)}$.

Las recesiones gingivales afectan a una significativa proporción de dientes por paciente ${ }^{(16)}$. Albandar en 1999 encuentra que el $58 \%$ de la población adulta estadounidense posee recesiones $\geq 1 \mathrm{~mm}^{(10)}$. Sarfati $2010^{(20)}$ encuentra un $84,6 \%$ de recesiones vestibulares en Francia. Susin $2004^{(6)}$ encuentra recesiones $\geq 3 \mathrm{~mm}$ en más de la mitad de los pacientes en Porto Alegre, Brasil. Rios et al., en 2014, encontraron una alta prevalencia de recesiones gingivales en una población brasileña. Nuestro estudio encontró una prevalencia de recesiones del 94,3\% y una extensión del $44,8 \%$, altas tasas también fueron encontradas por Toker y Ozdemir $2009^{(21)}$, con una prevalencia de recesiones del $78,2 \%$ en una población turca y por Raetzke ${ }^{(22)}$ con una prevalencia del $76-87 \%$ en una población alemana.

El aumento de la edad, el género masculino, fumar y la cantidad de placa bacteriana fueron indicadores de riesgo para las recesiones

Figura 2. Cantidad promedio de recesiones gingivales según el género y los rangos de edad evaluados. gr2 
Tabla 2. Distribución de los pacientes acorde a la presencia/ausencia de recesiones gingivales

\begin{tabular}{|c|c|c|c|c|c|c|c|c|c|c|}
\hline & \multicolumn{2}{|c|}{ Sin recesión } & \multicolumn{2}{|c|}{$R G \geq 1 \mathrm{~mm}$ en $\geq 1$ diente } & \multicolumn{2}{|c|}{$R G \geq 3 m m \geq 1$ diente } & \multicolumn{2}{|c|}{$\mathrm{RG} \geq 5 \mathrm{~mm} \geq 1$ diente } & \multicolumn{2}{|c|}{ Total } \\
\hline & $\mathbf{N}$ & $\%$ & $\mathbf{n}$ & $\%$ & $\mathrm{n}$ & $\%$ & $\mathrm{n}$ & $\%$ & $\mathbf{n}$ & $\%$ \\
\hline \multicolumn{11}{|l|}{ Edad } \\
\hline $20-29$ & 12 & 3,8 & 44 & 13,8 & 6 & 1,9 & 0 & 0 & 34 & 10,7 \\
\hline $30-39$ & 0 & 0 & 40 & 12,6 & 12 & 3,8 & 2 & 0,6 & 40 & 12,6 \\
\hline $40-49$ & 0 & 0 & 64 & 20,1 & 34 & 10,7 & 14 & 4,4 & 66 & 20,8 \\
\hline $50-59$ & 2 & 0,6 & 84 & 26,4 & 52 & 16,4 & 20 & 6,3 & 82 & 25,8 \\
\hline $60-69$ & 4 & 1,3 & 60 & 18,9 & 46 & 14,5 & 14 & 4,4 & 58 & 18,2 \\
\hline$\geq 70$ & 0 & 0 & 20 & 6,3 & 16 & 5 & 8 & 2,5 & 20 & 6,3 \\
\hline \multicolumn{11}{|l|}{ Género } \\
\hline Masculino & 6 & 1,9 & 146 & 45,9 & 88 & 27,7 & 36 & 11,3 & 144 & 45,3 \\
\hline Femenino & 12 & 3.8 & 166 & 52,2 & 78 & 24,5 & 22 & 6,9 & 156 & 49,1 \\
\hline \multicolumn{11}{|l|}{ Nivel de educación } \\
\hline Baja & 0 & 0 & 14 & 4,4 & 8 & 2,5 & 2 & 0,6 & 14 & 4,4 \\
\hline Media & 10 & 3,1 & 120 & 37,7 & 64 & 20,1 & 26 & 8,2 & 114 & 35,8 \\
\hline Alta & 8 & 2,5 & 178 & 56 & 94 & 29,6 & 30 & 9,4 & 172 & 54,1 \\
\hline \multicolumn{11}{|c|}{ Estado socioeconómico } \\
\hline Bajo & 2 & 0,6 & 54 & 17 & 40 & 12,6 & 18 & 5,7 & 52 & 16,4 \\
\hline Medio & 16 & 5 & 240 & 75,5 & 116 & 36,5 & 40 & 12,6 & 230 & 72,3 \\
\hline Alto & 0 & 0 & 18 & 5,7 & 10 & 3,1 & 0 & 0 & 18 & 5,7 \\
\hline \multicolumn{11}{|c|}{ Frecuencia de cepillado } \\
\hline$\leq 1 /$ día & 2 & 0,6 & 36 & 11,3 & 18 & 5,7 & 2 & 0,6 & 34 & 10,7 \\
\hline 2/día & 8 & 2,5 & 164 & 51,6 & 98 & 30,8 & 44 & 13,8 & 160 & 50,3 \\
\hline$\geq 3 /$ día & 8 & 2,5 & 112 & 35,2 & 50 & 15,7 & 12 & 3,8 & 106 & 33,3 \\
\hline \multicolumn{11}{|l|}{ Uso de hilo dental } \\
\hline Nunca & 16 & 5,1 & 240 & 75,9 & 128 & 40,5 & 52 & 16,5 & 228 & 72,2 \\
\hline$\leq 1 /$ día & 2 & 0,6 & 68 & 21,5 & 34 & 10,8 & 6 & 1,9 & 68 & 21,5 \\
\hline$\geq 2 /$ día & 0 & 0 & 2 & 0,6 & 2 & 0,6 & 0 & 0 & 2 & 0,6 \\
\hline \multicolumn{11}{|c|}{ Movimiento del cepillado } \\
\hline Horizontal & 6 & 1,9 & 106 & 33,3 & 50 & 15,7 & 16 & 5 & 102 & 32,1 \\
\hline Vertical & 6 & 1,9 & 116 & 36,5 & 72 & 22,6 & 30 & 9,4 & 112 & 35,2 \\
\hline Circular & 2 & 0,6 & 28 & 8,8 & 10 & 3,1 & 2 & 0,6 & 26 & 8,2 \\
\hline Todos & 4 & 1,3 & 62 & 19,5 & 34 & 10,7 & 10 & 3,1 & 60 & 18,9 \\
\hline \multicolumn{11}{|l|}{ Exposición al tabaco } \\
\hline No fuma & 16 & 5 & 270 & 84,9 & 146 & 45,9 & 50 & 15,7 & 260 & 81,8 \\
\hline Fumador moderado & 0 & 0 & 38 & 11,9 & 16 & 5 & 6 & 1,9 & 38 & 11,9 \\
\hline Fumador fuerte & 2 & 0,6 & 4 & 1,3 & 4 & 1,3 & 2 & 0,6 & 2 & 0,6 \\
\hline \multicolumn{11}{|l|}{ Visita dental } \\
\hline Nunca & 2 & 0,6 & 6 & 1,9 & 0 & 0 & 0 & 0 & 4 & 1,3 \\
\hline Irregular & 16 & 5 & 284 & 89,3 & 160 & 50,3 & 58 & 18,2 & 274 & 86,2 \\
\hline Regular & 0 & 0 & 22 & 6,9 & 6 & 1,9 & 0 & 0 & 22 & 6,9 \\
\hline \multicolumn{11}{|c|}{ Tratamiento periodontal previo } \\
\hline Sí & 0 & 0 & 58 & 18,2 & 32 & 10,1 & 6 & 1,9 & 58 & 18,2 \\
\hline No & 18 & 5,7 & 254 & 79,9 & 134 & 42,1 & 52 & 16,4 & 242 & 76,1 \\
\hline \multicolumn{11}{|c|}{ Tratamiento ortodóncico previo } \\
\hline Sí & 0 & 0 & 16 & 5 & 2 & 0,6 & 0 & 0 & 16 & 5 \\
\hline No & 18 & 5,7 & 296 & 93,1 & 164 & 51,6 & 58 & 18,2 & 284 & 89,3 \\
\hline
\end{tabular}

138 | Rev. Clin. Periodoncia Implantol. Rehabil. Oral Vol. 10(3); 135-140, 2017. 
Tabla 3. Modelo de regresión logística multivariable para los indicadores de riesgo de recesiones gingivales vestibulares

\begin{tabular}{|c|c|c|c|c|c|c|}
\hline & \multicolumn{6}{|c|}{ Recesiones gingivales vestibulares } \\
\hline & \multicolumn{2}{|c|}{$\geq 1-2 \mathrm{~mm}$} & \multicolumn{2}{|c|}{$\geq 3-5 \mathrm{~mm}$} & \multicolumn{2}{|c|}{$\geq 5 \mathrm{~mm}$} \\
\hline & OR & IC $95 \%$ & OR & IC $95 \%$ & OR & IC $95 \%$ \\
\hline \multicolumn{7}{|l|}{ Género } \\
\hline Masculino & 0,44 & $0,08-2,43$ & $1.6^{* *}$ & $1,05-2,5$ & $2^{*}$ & $1,09-3,68$ \\
\hline \multicolumn{7}{|l|}{ Femenino } \\
\hline \multicolumn{7}{|l|}{ Educación } \\
\hline Alta & Ref & & Ref & & Ref & \\
\hline Baja & & & 1,37 & $0,415-4,17$ & 0,7 & $0,14-3,79$ \\
\hline \multicolumn{7}{|l|}{ Media } \\
\hline \multicolumn{7}{|l|}{ Estado socioeconómico } \\
\hline Alto & Ref & & Ref & & Ref & \\
\hline Medio & & & 0,499 & $0,18-1,3$ & & \\
\hline Bajo & 1,6 & $0-0,01$ & 1,6 & $0,54-5,1$ & & \\
\hline \multicolumn{7}{|l|}{ Frecuencia del cepillado } \\
\hline$\leq 1 /$ día & & & 1,45 & $0,67-3,15$ & 0,42 & $0,08-2,08$ \\
\hline 2/día & 0,85 & $0,14-4,92$ & 1,48 & $0,89-2,48$ & 2,04 & $0,97-4,29$ \\
\hline$\geq 3 /$ día & Ref & & Ref & & Ref & \\
\hline \multicolumn{7}{|c|}{ Movimiento del cepillado } \\
\hline Horizontal & 1,73 & $0,23-12,7$ & 0,701 & $0,36-1,35$ & 1,47 & $0,55-3,87$ \\
\hline Vertical & 2,14 & $0,28-15,9$ & 1,19 & $0,62-2,28$ & 1,87 & $0,75-4,65$ \\
\hline Circular & & & $0,51^{*}$ & $1,19-1,35$ & $0,78^{*}$ & $1,14-4,27$ \\
\hline Todos & Ref & & Ref & & Ref & \\
\hline \multicolumn{7}{|l|}{ Visita dental } \\
\hline \multicolumn{7}{|l|}{ Nunca } \\
\hline Irregular & & & 2,36 & $0,89-6,25$ & & \\
\hline Regular & Ref & & Ref & & Ref & \\
\hline \multicolumn{7}{|l|}{ Exposición al tabaco } \\
\hline No fuma & & & & & 0,39 & $0,05-2,92$ \\
\hline Fumador moderado & 1,25 & 1,25 & & & 0,28 & $0,03-2,71$ \\
\hline Fumador fuerte & Ref & & Ref & & Ref & \\
\hline \multicolumn{7}{|c|}{ Tratamiento periodontal previo } \\
\hline Sí & 1,02 & $1-1,04$ & 1,15 & $0,65-2,05$ & 0,53 & $0,21-1,3$ \\
\hline No & Ref & & Ref & & Ref & \\
\hline \multicolumn{7}{|c|}{ Tratamiento ortodóncico previo } \\
\hline Sí & 1,02 & $1-1,03$ & 1,12 & $0,02-0,53$ & $1,2^{*}$ & $1,14-1,27$ \\
\hline No & Ref & & Ref & & Ref & \\
\hline
\end{tabular}

gingivales ${ }^{(16)}$. Rios et al. ${ }^{(16)}$ encuentran mayores tasas de RG en varones que en mujeres; Susin6 no encuentra el género como indicador de riesgo. Rios et al. ${ }^{(16)}$ encuentran mayor odds ratio entre recesiones $\geq 3 \mathrm{~mm}$ y la exposición al tabaco. Es posible por el efecto perjudicial del tabaco sobre el periodonto marginal ${ }^{(16)}$. Nuestro estudio encontró una significativa asociación entre el género masculino y una mayor severidad de recesiones gingivales $(O R=2, I C: 1,09-3,68)$, sin embargo, el género femenino evidenció mayor prevalencia de recesiones gingivales $(49,1 \%$ vs $45,3 \%$ ); Kozlowska et al. ${ }^{(23)}$ encuentran más recesiones en mujeres que en varones $-31,7 \%$ vs $24,3 \%$-; esto puede explicarse debido a que las mujeres se encuentran más motivadas con el cuidado de la higiene oral y poseen mayores frecuencias de cepillado que los varones ${ }^{(24)}$. La exposición al tabaco evidenció una asociación no significativa con respecto a la severidad de las recesiones gingivales $(O R=0,28, I C: 0,03-$ 2,71).

Las recesiones gingivales se han asociado a bajos niveles de higiene oral. Individuos con un mayor porcentaje de sangrado gingival tuvieron recesiones gingivales más severas ${ }^{(16)}$. Las visitas dentales regulares y el tratamiento periodontal previo también tuvieron grandes chances de tener recesiones gingivales ${ }^{(16)}$. Nuestro datos no encontraron una asociación significativa entre las cantidad de visitas dentales y la severidad de recesiones gingivales $(\mathrm{OR}=2,36, \mathrm{IC}=0,89-6,25)$. 
Si bien se encontró una alta prevalencia de recesiones gingivales, el estudio con diseño transversal no permite realizar un inferencia inequívoca causal de los indicadores de riesgo y recesiones gingivales: son necesarios los estudios de cohortes para establecer una verdadera relación de causalidad. La presente investigación recabó información de una población urbana, una muestra que posee acceso a los servicios odontológicos; es necesario conocer datos de poblaciones rurales muestras más extensas e incluir dentro de los datos a evaluar el impacto de las recesiones gingivales en la calidad de vida de los pacientes. La calidad de vida asociada a la salud bucal es más baja en la población adulta con recesiones gingivales, principalmente cuando se ubican en dientes anterosuperiores ${ }^{(25)}$. La hipersensibilidad dentinaria y la estética son 2 factores asociados a las recesiones gingivales sobre la calidad de $\operatorname{vida}^{(25)}$. Los individuos con $\geq 1$ diente con $\mathrm{GR} \geq 2 \mathrm{~mm}$ tenían aproximadamente 2 veces mayor probabilidad de tener un impacto negativo que las personas sin GR de este umbral [odds ratio=1,99, IC 95\%: $1,05-3,78]^{(25)}$.

Concluimos que la prevalencia de recesiones gingivales en una muestra peruana es elevada (94\%), la extensión es medianamente alta (44\%), siendo las piezas dentarias mandibulares las más afectadas; la severidad promedio de las recesiones fue de $3,86 \pm 0,45 \mathrm{~mm}$. Se encontraron asociaciones entre el género, el nivel de educación, el estado socioeconómico, la frecuencia del cepillado, el movimiento de cepillado, las visitas dentales, la exposición al tabaco y el tratamiento ortodóncico previo; sin embargo, solo fueron significativos el género, el movimiento del cepillado y el tratamiento ortodóncico con respecto a la severidad de las recesiones gingivales.

\section{RESPONSABILIDADES ÉTICAS}

\section{Protección de personas y animales}

Los autores declaran que los procedimientos seguidos se conformaron a las normas éticas del comité de experimentación humana responsable y de acuerdo con la Asociación Médica Mundial y la Declaración de Helsinki.

\section{Confidencialidad de los datos}

Los autores declaran que han seguido los protocolos de su centro de trabajo sobre la publicación de datos de pacientes.

Derecho a la privacidad y consentimiento informado

Los autores han obtenido el consentimiento informado de los pacientes y/o sujetos referidos en el artículo. Este documento obra en poder del autor de correspondencia.

\section{CONFLICTO DE INTERESES}

Los autores no mostramos ningún tipo de conflicto de intereses con respecto al artículo.

\section{Bibliografía}

1. Chambrone L, Sukekava F, Araujo M. Root coverage procedures for treatment of localized recession type defects a Cochrane systematic review. J Periodontol. 2010;81:452-78

2. Cortellini P, Pini-Prato G. Coronally advanced flap and combination therapy for root coverage. Clinical strategies based onscientific evidence and clinical experience. Periodontol 2000.2012:59:158-84

3. Castro $Y$, Grados S. Porcine collagen matrix for treating gingival recession. Randomized clinical trial. J Oral Res. 2014;3:23-8.

4. Richardson CR, Allen EP, Chambrone L, Langer B, McGure MK,Zabalegui I, et al. Periodontal soft tissue root coverage procedures: Practical applications from the AAP regeneration workshop.Clin Adv Periodontics. 2015;5:2-10.

5. Maetahara R. Prevalencia, extensión y severidad de las recesiones gingivales en pacientes del Hospital Central de la Fuerza Aérea del Perú [tesis para optar el título de cirujano dentista]. [Lima]: Universidad Nacional Mayor de San Marcos; 2006.p. 110.

6. Susin C, Haas AN, Oppermann RV, Haugejorden O, AlbandarJM. Gingival recession: Epidemiology and risk indicators in a representative urban Brazilian population. J Periodontol.2004;75:1377-86.

7. O'Leary TJ, Drake RB, Jividen GJ, Allen MF. The incidence ofrecession in young males: Relationship to gingival and plaque scores. Periodontics. 1969;6:109.

8. Gorman WJ. Prevalence and etiology of gingival recession. J Periodontol. 1967;38:316.

9. Albandar JM, Kingman A. Gingival recession, gingival bleeding, and dental calculus in adults 30 years of age and older in the United States, 1988-1994. J Periodontol. 1999;70:30-43.

10. Novaes A, Novaes Jr. Cirugía periodontal con finalidad protésica. Brasilia. 1.a ed. Artes médicas; 2001. p. 96

11. Kassab M, Cohen R. The etiology and prevalence of gingival recession. J Am Dent Assoc. 2003;134:220-5

12. Tugnait A, Clerehugh V. Gingival recession, its significance and management. J Dent. 2001:29:381-94.

13. Pires IL, Cota LO, Oliveira AC, Costa JE, Costa FO. Association between periodontal condition and use of tongue piercing: Acase-control study. J Clin Periodontol. 2010;37:712-8.
14. Dilsiz A, Aydin T Gingival recession associated with orthodontic treatment and root coverage. J Clin Exp Dent. 2010;2:e30-3.

15. Loe $\mathrm{H}$, Anerud A, Boysen $\mathrm{H}$. The natural history of periodontal disease in man prevalence, severity, and extent of gingival recession. J Periodontol. 1992;63:489-95. 16. Rios FS, Costa RSA, Moura MS, Jardim JJ, Maltz M, Haas AN.Estimates and multivariable risk assessment of gingival recession in the population of adults from Porto Alegre, Brazil. J Clin Periodontol. 2014;41:1098-107.

17. ABEP (2013). Brazilian association of research companies-Criterio de classificacao economica Brasil (CCEB) [consultado 3 Abr 2016]. Disponible en: http://www.abep.org/novo/Content.aspx?ContentID=835

18. Heasman PA, Holliday R, Bryant A, Preshaw PM. Evidence for the occurrence of gingival recession and non-carious cervical lesions as a consequence of traumatic toothbrushing. J Clin Periodontol. 2015;42 Suppl 16:S237-55.

19. Khocht A, Simon G, Person P, Denepitiya JL. Gingival recessionin relation to history of hard toothbrush use. J Periodontol.1993;64:900-5.

20. Sarfati A, Bourgeois D, Katsahian S, Mora F, Bouchard P. Risk assessment for buccal gingival recession defects in an adult population. J Periodontol. 2010;81:1419-25

21. Toker H, Ozdemir H. Gingival recession: Epidemiology and risk indicators in a university dental hospital in Turkey. Int J Dent Hygiene. 2009:115-20.

22. Raetzke R. Parodontale rezession-pravelenz, signifikanz, ursachen and therapie. Zanhnarztl Welt. 1985;94:968-71.

23. Kozlowska M, Wawrzyn-Sobczak K, Karczewski JK, StokowskaW. The oral cavity hygiene as the basic element of the gingival recession prophylaxis. Rocz Akad Medyc Bial Mstok.2005:50:234-7.

24. Mumghamba EG, Honkala S. Gingival recession, oral hygiene and associated factors among Tanzanian women. East Afr Med J.2009;86:125-32.

25. Wagner TP, Costa RSA, Rios FS, Moura MS, Maltz M, Jardim JJ, et al. Gingival recession and oral health-related quality of life: A population-based cross-sectional study in Brazil. Community Dent Oral Epidemiol. 2016;44:390-9. 